\title{
Exploring the full natural diversity of single amino acid exchange reveals that 40- $60 \%$ of BSLA positions improve organic solvents resistance
}

\author{
Victorine Josiane Frauenkron-Machedjou' ${ }^{1}$, Alexander Fulton ${ }^{4}$, Jing Zhao ${ }^{1,2}$, Lina Weber ${ }^{1}$, Karl-Erich Jaeger ${ }^{4,5}$, \\ Ulrich Schwaneberg ${ }^{1,3}$ and Leilei Zhu ${ }^{1,2^{*}}$
}

\begin{abstract}
Objectives: Protein engineering has been employed to successfully improve organic solvent resistance of enzymes. Exploration of nature's full potential (how many beneficial positions/beneficial substitutions of the target enzyme) to improve organic solvent resistance of enzymes by a systematic study was performed.

Results: We report the results of screening the previously generated BSLA (Bacillus subtilis lipase A)-SSM (site saturation mutagenesis) library (covering the full natural diversity of BSLA with one amino acid exchange) in presence of three cosolvents. The potential of single amino acid substitution that nature offers to improve the cosolvent resistance of BSLA was determined by analyzing the number of beneficial positions/substitutions, accessibility and chemical compositions.

Conclusion: Lessons learned from analysis of BSLA-SSM library are: (1) 41-59\% of BSLA positions with in total 4-10\% of all possible substitutions improve the cosolvent resistance against TFE, DOx, and DMSO; (2) charged substitutions are preferred to improve DOx and TFE resistance whereas polar ones are preferred for DMSO; (3) charged substitutions on the surface predominantly improved resistance while polar ones were preferred in buried "regions". (4) Interestingly, 58-93\% of beneficial substitutions led to chemically different amino acids.
\end{abstract}

Keywords: BSLA, Directed evolution, Protein engineering, Site saturation mutagenesis, Organic solvents resistance

\section{Background}

Being able to routinely reengineer enzymes for efficient enzymatic catalysis in organic solvents would be highly attractive for chemical production in general and overcome limitations of biocatalysis in water such as limited solubility of hydrophobic substrates and products, suppression of unwanted side reactions, cost-effective product recovery and thereby avoiding waste water treatment (Arnold 1990; Castro and Knubovets 2003). General principles to redesign and to stabilize enzymes in organic

\footnotetext{
*Correspondence: zhu_II@tib.cas.cn

${ }^{2}$ Tianjin Institute of Industrial Biotechnology, Chinese Academy of Sciences, West 7th Avenue 32, Tianjin 300308, China Full list of author information is available at the end of the article
}

solvents or cosolvents are therefore of high importance and first molecular insights have been discovered (Micaelo and Soares 2007; Li et al. 2010). Enzymes maintain their structural conformations through intramolecular interactions among amino acids and between amino acid and surrounding water molecules. A prerequisite to maintain structural integrity, flexibility and activity in non-aqueous solvents or cosolvent mixtures is that water molecules remain bound to the enzyme to ensure its flexibility (Zaks and Klibanov 1988). Hydrophilic organic solvents (e.g. EtOH, $\mathrm{MeOH}$, and DMSO) with a polarity similar to water are fully miscible (homogeneous solution) (Park et al. 2012) and organic molecules are in close contact with enzymes. The latter can result in a 
competition of solvent molecules with enzyme-bound water molecules and results in structural changes or denaturation (Ogino and Ishikawa 2001; Yang et al. 2004). In contrast, hydrophobic solvents (e.g. heptane, hexane and toluene) exhibit poor solubility in water and compete much less for enzyme-bound water molecules. Therefore, hydrophobic solvents are often unable to remove substantial amounts of water from enzymes (Gorman and Dordick 1992), and often do not affect enzyme activity. In few cases, it was reported that organic solvent molecules can reduce enzymatic activities without altering the enzyme structure for instance by competing with the sixth water ligand of P450 BM3 at the catalytic heme center (Roccatano et al. 2006; Wong et al. 2004).

Enzyme immobilization and supplements of stabilizers (such as salts, sugars, surfactants) have been reported to effectively stabilize enzymes in presence of organic solvents (Stepankova et al. 2013). In the last two decades altering the enzyme stability by protein engineering developed into a powerful alternative to overcome limitations in solvent resistance (Stepankova et al. 2013; Zhao et al. 2015; Markel et al. 2017). Overall, more than 24 reports have been published in which (co-)solvent resistance has been improved ( $\geq 15$ reports by directed evolution; $\geq 9$ reports by semi-rational design). Till today, improved organic (co-)solvent resistance has been reported for at least four enzyme classes including monooxygenases, hydrolases, oxidoreductases, and transferases (Wong et al. 2004; Kawata and Ogino 2009, 2010; Koudelakova et al. 2013; Liu et al. 2009, 2013; Martinez and Arnold 1991). The reported beneficial amino acid substitutions provided the insight that how enzymes can be stabilizes organic (co-)solvents. For instance, it was found that substitutions of two charged amino acid residues on the surface of $\alpha$-lytic protease to hydrophobic residues improved protease stability in presence of hydrophilic solvents (Martinez and Arnold 1991). Interestingly, it was reported that some beneficial substitutions were surface exposed (Park et al. 2012, 2013; Kawata and Ogino 2009; Martinez et al. 1992). Furthermore, the formation of salt bridges and hydrogen bonds led to improved organic solvent stability of the lipase (Pseudomonas aeruginosa LST-03) in presence of organic solvents (Kawata and Ogino 2010). However, in the above-mentioned semi-rational design and traditional directed evolution experiments, a limited number of clones was screened (usually a few thousand) and resulted in less than 10 beneficial amino acids substitutions. Due to the limited number of screened variants, a comprehensive picture which includes all possible stabilization modes was not reported.

BSLA belongs to the lipase subfamily I-4, contains 181 amino acids in its mature form and shares $74-80 \%$ sequence identity with other members of this subfamily (Arpigny and Jaeger 1999; Bustos-Jaimes et al. 2010). An SSM library covering all natural diversity with a single amino acid exchange (named 'BSLA-SSM library') has been previously reported (Frauenkron-Machedjou et al. 2015; Fulton et al. 2015). The BSLA-SSM library mimics natural evolution, which evolves enzymes by introducing single amino acid exchanges due to its very low mutation frequency (Lynch 2010).

In this report, the potential that nature offers to improve the cosolvent resistance of BSLA was determined by screening the BSLA-SSM library against three cosolvents (TFE, DOx, and DMSO) and statistical analysis of beneficial substitutions. Analysis was performed by determining the number of amino acid positions and substitutions that increase BSLA's organic solvent resistance. Subsequently, amino acid substitution patterns were investigated in respect to accessibility (surface exposed and buried residues), chemical compositions of beneficial substitutions, and their location within structural elements. The latter analysis teaches lessons on how to stabilize BSLA in cosolvents and enables computational-assisted protein engineering for discovering general principles of cosolvent tolerance.

\section{Methods}

\section{Chemicals and reagents}

All chemicals were of analytical grade or higher and were purchased from Sigma Aldrich (Steinheim, Germany), AppliChem (Darmstadt, Germany), Fluka (NeuUlm, Germany), and Carl Roth (Karlsruhe, Germany). Enzymes and dNTPs were obtained from New England Biolabs (Frankfurt, Germany) and Sigma Aldrich (Steinheim, Germany). The HPLC-purified oligonucleotides applied for mutagenesis were obtained from GATC (Konstanz; Germany) and Eurofins MWG Operon (Ebersberg, Germany). The oligonucleotide sequences and growth media are summarized or described in Additional file 1 .

\section{Strains and plasmids}

The template for all PCRs was the plasmid pET22b(+)bsla WT. For DNA manipulation and recombinant protein expression E. coli DH5 $\alpha$ and E. coli BL21-Gold (DE3) (Agilent Technologies; Santa Clara, USA) were used as hosts for DNA manipulation and recombinant protein expression, respectively. Plasmid purification was performed employing the kit "NucleoSpin Plasmid kit" from Macherey-Nagel (Düren, Germany) according to the supplier's recommendations, and DNA concentrations were quantified using a NanoDrop photometer (NanoDrop Technologies, Germany). 


\section{Site saturation mutagenesis on the whole BSLA gene sequence}

SSM (Barettino et al. 1994) libraries targeting the whole bsla were generated using degenerated NNS $(\mathrm{N}=\mathrm{A} / \mathrm{T} /$ $\mathrm{G} / \mathrm{C}$ and $\mathrm{S}=\mathrm{C} / \mathrm{G}$ ) oligonucleotide primers encoding all 20 amino acids with 32 distinct codons (Additional file 1: Tables S1, S2) as described in our previous publication (Frauenkron-Machedjou et al. 2015).

\section{Cultivation and expression in 96-well plates}

After transformation of the PCR product of SSM libraries, single colonies were grown on $\mathrm{LB}_{\mathrm{Amp}}$ agar plates. For each SSM library, 92 BSLA clones were picked to $150 \mu \mathrm{L}$ $\mathrm{LB}_{\text {Amp }}$ medium in 96-well microtiter plate (MTP) (flatbottomed, polystyrene plates; Greiner Bio-One $\mathrm{GmbH}$, Frickenhausen, Germany). In addition, three BSLA WT clones and one empty vector clone were used as control in the same MTP. The samples were proceeded with as described previously (Frauenkron-Machedjou et al. 2015).

\section{pNPB assay in MTP for BSLA activity measurement}

$p$-Nitrophenyl butyrate $(p \mathrm{NPB})$ was used as substrate to detect the activity of BSLA (Shirai et al. 1982). BSLA converts $p$ NPB to $p$-nitrophenolate, which can be detected at $410 \mathrm{~nm}$. The activity of the assay was determined by addition of TEA buffer $(90 \mu \mathrm{L}, 50 \mathrm{mM}, \mathrm{pH} 7.4)$ to supernatant $(10 \mu \mathrm{L})$ and freshly prepared substrate solution $(100 \mu \mathrm{L})$ containing $p$ NPB $(0.5 \mathrm{mM})$ and acetonitrile $[10 \%(\mathrm{v} / \mathrm{v})]$ in each well. The release of $p$-nitrophenolate was recorded by measuring $\mathrm{A}_{410}$ at room temperature over $8 \mathrm{~min}$ on a microtiter plate reader (Tecan infinite ${ }^{\circledR}$ M200 Pro Austria $\mathrm{GmbH}$, Männedorf, Switzerland). The standard deviation of the assay was determined by measuring the activity in a 96-well MTP containing only BSLA WT and another 96-well MTP containing only EV. The apparent standard deviation was calculated without subtracting the background; the true standard deviation was obtained after background subtraction.

\section{Optimization of screening conditions in organic solvents}

DMSO, DOx and TFE were used in this work to screen the generated SSM-BSLA library. In order to determine the concentration of the three organic solvents for SSM libraries screening, the solvents were supplemented with TEA buffer (pKa 7.76, pH 7.4, $50 \mathrm{mM}$ ) to obtain organic solvents with different concentrations. All three organic solvents were water-miscible as described in the safety sheets provided by the suppliers Sigma-Aldrich (Steinheim, Germany) and Carl Roth (Karlsruhe, Germany). Besides, the $\mathrm{pH}$ of the incubated mixtures (lipase, buffer, organic solvent) as well as the $\mathrm{pH}$ of the reaction mixture after product formation was measured. $\mathrm{pH}$ shifts of
$<0.22 \mathrm{pH}$ units in the presence of organic solvents were observed. Concentrations leading to a residual activity between 30 and $40 \%$ of the BSLA WT were chosen to screen the SSM libraries.

\section{Screening of SSM-BSLA library in presence of the three organic solvents}

The screening assay with organic solvents was performed in polypropylene MTP (flat-bottomed, polypropylene plates; Greiner Bio-One $\mathrm{GmbH}$, Frickenhausen, Germany), which is resistant to 1,4-dioxane. In each well, supernatant $(10 \mu \mathrm{L})$ was incubated with the organic solvent solution $(90 \mu \mathrm{L}$, concentrations in Table 1$)$ or with buffer (control) for $2 \mathrm{~h}$ at RT on a microtiter shaker (1000 rpm, Edmund Bühler Microtiter shaker, Hechingen, Germany). Freshly prepared substrate solution (100 $\mu \mathrm{L} /$ well) was added and the $\mathrm{A}_{410}$ was measured at RT over 8 min (Tecan infinite ${ }^{\circledR}$ M200 Pro Austria GmbH, Männedorf, Switzerland). For each well, the same amount of supernatant was incubated with or without organic solvent. BSLA resistance (WT or variant) was evaluated as activity in the presence of organic solvent divided by activity in absence of organic solvent (equation in Additional file 1).

For all the analysis, the SSM-BSLA variants are identified by the following symbols: "+" variants with improved resistance $\left(R_{\mathrm{V}} \geq R_{\mathrm{WT}}+3 \sigma\right)$; "=" variants with unchanged resistance $\left(R_{\mathrm{V}}<R_{\mathrm{WT}}+3 \sigma\right.$ and $\left.\mathrm{R}_{\mathrm{V}}>\mathrm{R}_{\mathrm{WT}}-3 \sigma\right)$; “-” variants with decreased resistance $\left(R_{\mathrm{V}} \leq R_{\mathrm{WT}}-3 \sigma\right)$, and " $\times$ " variants shows non-detectable activity in buffer under assay condition $\left(A_{\mathrm{V}}<A_{\mathrm{EV}}+3 \sigma\right)$. $\mathrm{R}$ abbreviates residual activity (defined in equation 1 in Additional file 1 ), A: activity, V: variant, WT: wild type, EV: empty vector, and represents standard deviation of the screening system in presence of each organic solvent (see Table 1).

\section{Results}

The result section is divided in six parts. The first part deals with the conditions used for the screening of SSMBSLA library in presence of three organic solvents, and the second part focuses on the analysis of the number of amino acids positions which influence organic cosolvent resistance of BSLA (improved/decreased/unchanged organic solvents resistance and inactivation). In the third part, all substitutions were analyzed to determine the number of single amino acid substitutions that led to increased/unchanged/decreased BSLA resistance towards the three investigated organic cosolvents. In the fourth part, the amino acid substitutions were grouped into four types (aromatic, aliphatic, polar, and charged) and the influence of each type of amino acid on BSLA resistance was depicted. The fifth part focuses on the effect of substituting each type of amino acid with all the 
Table 1 Screening system of BSLA in three organic cosolvents

\begin{tabular}{llllr}
\hline Organic cosolvent & Conc. $(\mathbf{M})^{\mathbf{a}}$ & $\boldsymbol{R}_{\mathbf{W T}}(\%)$ & \multicolumn{2}{l}{$\begin{array}{l}\text { Standard devia- } \\
\text { tion }(\%)\end{array}$} \\
\cline { 3 - 5 } & $(\% \mathbf{~ v / v )}$ & & Apparent & True \\
\hline DMSO & $8.4(\approx 60.0)$ & 29.0 & 8.0 & 10.5 \\
DOx & $2.6(\approx 22.0)$ & 31.0 & 6.5 & 9.6 \\
TFE & $2.9(\approx 12.0)$ & 30.0 & 7.6 & 12.0 \\
\hline
\end{tabular}

Conc. concentration, $R_{W T}$ residual activity of BSLA WT. Apparent and true standard deviation values for BSLA WT in the respective organic cosolvents, DMSO dimethyl sulfoxide, DOx 1,4-dioxane, TFE 2,2,2-trifluoroethanol

${ }^{a}$ Concentration used in the incubation step for the BSLA activity assay

four amino acid types and the concluding part summarizes the role that location of amino acid substitutions has on organic solvent resistance of BSLA.

\section{Condition used for screening of SSM-BSLA library in presence of three organic solvents}

The SSM-BSLA library is the one previously described (Frauenkron-Machedjou et al. 2015) and contains 3620 possible single variants. All SSM-BSLA variants were screened in presence of three organic cosolvents (DMSO, DOx and TFE). The concentration of the three organic solvents used for the screening system was selected to obtain a residual activity of $30-40 \%$ of the BSLA WT (Table 1). True standard deviations of $9.6-12.0 \%$ were obtained for the BSLA screening systems with the three organic cosolvents. Screening systems with true coefficient of correlations around $12 \%$ were commonly used in directed evolution experiments (Wong et al. 2007).

After screening of the SSM-BSLA library in buffer and in presence of each of the three organic solvents, residual activity of BSLA variants $\left(R_{\mathrm{V}}\right)$ and the residual activity of WT $\left(R_{\mathrm{WT}}\right)$ were calculated. Based on the Gaussian distribution curve ( $\sigma$ is the true coefficient of correlation of the screening system see Table 1), all the variants were grouped into four categories as described previously in this report.

\section{Number of amino acid positions which influence organic solvent resistance of BSLA}

Figure 1 depicts the number of amino acid positions of BSLA, which led to improved/unchanged/decreased organic solvent resistance as well as inactivation of BSLA. For the organic solvent resistance type "+", it was found that DMSO (107 positions; 59\%) yields 32 more positions than 1,4 -dioxane $(75 ; 41 \%)$ and 33 positions more than TFE $(74 ; 41 \%)$, respectively. For the organic solvent resistance type "=", at each of the 181 amino acid positions at least one amino acid substitution was found that did not influence organic solvent resistance of BSLA. For the organic solvent resistance type "-", it was found that 1,4-dioxane (136, 75\% of all positions) yields more positions than TFE $(120 ; 66 \%)$ and DMSO (118; 65\%). For the resistance type " $x$ ", at least one amino acid substitution representing the wild type amino acid was found at 99 (55\%) amino acid positions, which inactivated BSLA already in the buffer solution. Detailed analysis of the number of substitutions at each position of BSLA as well as the overall number of amino acid substitutions on secondary elements of BSLA leading to improved/ decreased/un-changed organic solvent resistance and inactivation are given in Additional file 1: Tables S3-S6. In addition, 3D models of BSLA for visualization of the beneficial positions for all three organic solvents are illustrated in Additional file 1: Figures S1-S3.

\section{Number of single amino acid substitutions which affect organic solvent resistance of BSLA}

Figure 2 shows the number of variants with improved “+”, unchanged "=", decreased "-" resistance in presence of DMSO, DOx, and TFE as well as "inactive" variants " $x$ ".

For the organic solvent resistance type "+", it was observed that DMSO (371 out of $3620,10.2 \%$ of all substitutions) yields 190 substitutions more than TFE (181, $5.0 \%)$ and 212 more than DOx $(159,4.4 \%)$. Among the variants with increased resistance towards TFE, $0.17 \%$ showed a resistance improvement higher than 2.5fold, which is 6-fold higher than the values obtained for DMSO and DOx (0.03\%). Overall, 61.1-63.2\% (22122288 out of 3620 ) of all the substitutions had no effect on BSLA resistance type "=" towards the three selected organic cosolvents. For the organic cosolvent resistance type "-", similar results were achieved in DOx (675 out of $3620,18.6 \%$ of all substitutions) and TFE $(664,18.3 \%)$ which are 136 and 125 substitutions more than in DMSO (539, 14.9\%). For the type “ $\times$ ", 498 (13.7\%) substitutions resulted in inactivation of BSLA.

\section{The influence of amino acid "types" (aromatic, aliphatic, charged, polar) on BSLA's organic solvent resistance}

The 20 amino acid substitutions were grouped into four "types" in order to be consistent with previous reports (Wong et al. 2007, 2006; Verma et al. 2014). 15\% aromatic (F, Y, and W), 25\% aliphatic (A, V, L, I and G), $25 \%$ charged (D, E, H, K and R) and $35 \%$ polar (termed neutral in the cited papers; $C, M, P, S, T, N$ and $Q$ ) amino acids. Cysteine $(\mathrm{C})$, glycine $(\mathrm{G})$, and proline $(\mathrm{P})$ could also be classified as special amino acids. Figure 3 illustrates the effect of each type of amino acid substitution on the resistance of BSLA towards the three organic cosolvents. Inactive variants were defined as variants which showed no detectable activity in buffer. 




Fig. 1 Number of amino acid positions which lead to improved/unchanged/decreased organic solvent resistance and inactivation of BSLA in buffer. + : variants with improved resistance; $=$ : unchanged resistance; - : decreased resistance; $\times$ : inactive in buffer (see text)

Supplementing of organic solvent did not in single case lead to regain BSLA activity. In total, 183 out of 498 inactive BSLA variants (" $x$ " type in buffer) harbored charged (37\%), 152 polar (31\%), 87 aromatic (17\%), and 76 aliphatic substitutions (15\%).

Figure $3 \mathrm{a}-\mathrm{c}$ show the number of variants displaying the resistance type "+" towards DMSO; out of 371 beneficial substitutions 126 were to polar (34\%), 117 to charged (32\%), 88 to aliphatic (24\%), and 40 to aromatic amino acids (11\%). In case of DOx the 159 beneficial substitutions were composed of 62 to charged (39\%), 47 to polar (30\%), 26 to aliphatic (16\%), and 24 to aromatic amino acids (15\%). 181 beneficial substitutions were obtained for TFE. 62 to charged (34\%), 57 to polar (31\%), 34 to aromatic (19\%), and 28 to aliphatic substitutions (15\%). Overall, BSLA variants with substitutions to charged amino acids were the majority and therefore preferred type of substitutions to improve organic cosolvents' resistance of BSLA towards TFE and DOx. In case of DMSO, substitutions to polar amino acids were the preferred substitution type. The highest number of improved BSLA variants was obtained for DMSO (126) followed by DOx (62) and TFE (62) (Fig. 3a-c).

For the organic solvent resistance type "=", a similar trend of amino acid substitution was observed for all three organic solvents (36\% to polar, $28-29 \%$ to aliphatic, $20-22 \%$ to charged, and $14 \%$ to aromatic substitutions; Additional file 1: Table S7).
The organic solvent resistance type "-" for DMSO is as follows: from 539 beneficial substitutions, 197 were to polar (37\%), 127 to charged (24\%), 111 to aliphatic (21\%), and 104 to aromatic amino acids (19\%); for DOx, 242 out of 675 beneficial substitutions were to polar (36\%), 191 to charged (28\%), 132 to aliphatic (20\%), and 110 to aromatic amino acids (16\%); for TFE, 230 out of 664 beneficial substitutions were to polar (35\%), 181 to charged (27\%), 153 to aliphatic (23\%), and 100 to aromatic amino acids (15\%). Remarkably, BSLA variants with polar amino acid substitutions largely led to a decrease in cosolvent resistance for all three organic solvents; additionally significant differences in the total number of beneficial substitutions were observed (539 substitutions in DMSO vs 675 in DOx).

For DMSO (Fig. 3a), the number of beneficial substitutions are in order polar $>$ charged $>$ aliphatic $>$ aromatic. A different ranking was observed for DOx (Fig. 3b; charged $>$ polar $>$ aliphatic $\geq$ aromatic) and TFE (Fig. 3c; charged $>$ polar $>$ aromatic $>$ aliphatic). For the variants with organic solvent resistance type "=", the following order was observed: polar $>$ aliphatic $>$ charged $>$ aromatic for all three organic cosolvents. The number of variants for the resistance type "-" followed the order: polar > charged > aliphatic > aromatic in presence of all three organic solvents. In summary, the main type of beneficial substitutions differs from DOx and TFE (charged preferred) to DMSO (polar preferred). 




Fig. 2 Number of SSM-BSLA variants which lead to improved/unchanged/decreased organic solvent resistance and inactivation of BSLA in buffer. + : variants with improved resistance; $=$ : unchanged resistance; - : decreased resistance; $\mathbf{x}$ : inactive in buffer (see text)

Influence of amino acid substitution types on organic solvent resistance of BSLA: comparison between WT and introduced substitutions

Table 2 summarizes, in a matrix for three cosolvents (TFE, DOx, DMSO) and four substitution types (polar, aliphatic, charged and aromatic), the results on the chemical nature of substitutions patterns to which types of amino acid substitutions are more preferential. For all the three selected organic cosolvents, charged and polar substitutions are the preferred substitution types, except for the case of substituting aromatic ones towards DMSO and DOx. Another trend is that mainly the substitutions to chemically different amino acids led to improved organic solvent resistance with significant differences in preference, except for charged amino acid in BSLA WT. The following paragraphs analyze the substitutions patterns (substitutions to) of polar, aliphatic, charged and aromatic amino acids in respect to the three cosolvents.

When substituting the 15 aromatic amino acids in BSLA WT, 44, 19 and 12 beneficial substitutions were obtained for DMSO, DOx and TFE, respectively. Among them, the two most preferred amino acid substitutions are either charged/aliphatic (for DMSO and DOx) or charged/polar for TFE. Substituting the 79 aliphatic amino acids resulted in 155, 60 and 69 beneficial substitutions for DMSO, DOx and TFE, respectively. The two most favorable amino acid substitutions are charged and aliphatic for all the three selected organic cosolvents. Substituting the 54 polar amino acids led to 108, 56 and 62 beneficial substitutions were obtained for DMSO,
DOx and TFE, respectively. The two most preferred amino acid substitutions for all the three selected organic cosolvents are charged and polar. When substituting the 33 charged amino acids, 64, 24 and 38 beneficial substitutions were obtained for DMSO, DOx and TFE, respectively. Among them, the two most preferred amino acid substitutions are also charged and polar for all the three selected organic cosolvents.

Additional file 1: Tables S8, S9 summarize effects of positively charged and negatively charged amino acids.

\section{Effect of the location of amino acid substitution on organic solvent resistance of BSLA}

Around $71 \%$ amino acid residues of BSLA are surface exposed and 29\% are buried (Table 3). The solvent accessible surface for each residue was calculated using the Accelrys Discover Studio software package employing a water probe with a radius of $1.5 \AA$ and a cutoff of $<5$ solvent accessibility for buried residues (Chothia 1976) based on the chain A of the BSLA X-ray crystal structure (PDB ID: 1I6 W) (van Pouderoyen et al. 2001).

Both exposed and buried amino acid positions led to improved organic solvent resistance ("+") for all the four types of substitutions. At exposed positions, more beneficial substitutions were obtained than at buried positions for all the three organic cosolvents. On average, there are 1.1-2.1 beneficial substitutions per exposed position but only $0.4-1.8$ beneficial substitutions per buried position.

At exposed positions, the highest number of improved variants (type "+") was obtained with charged 

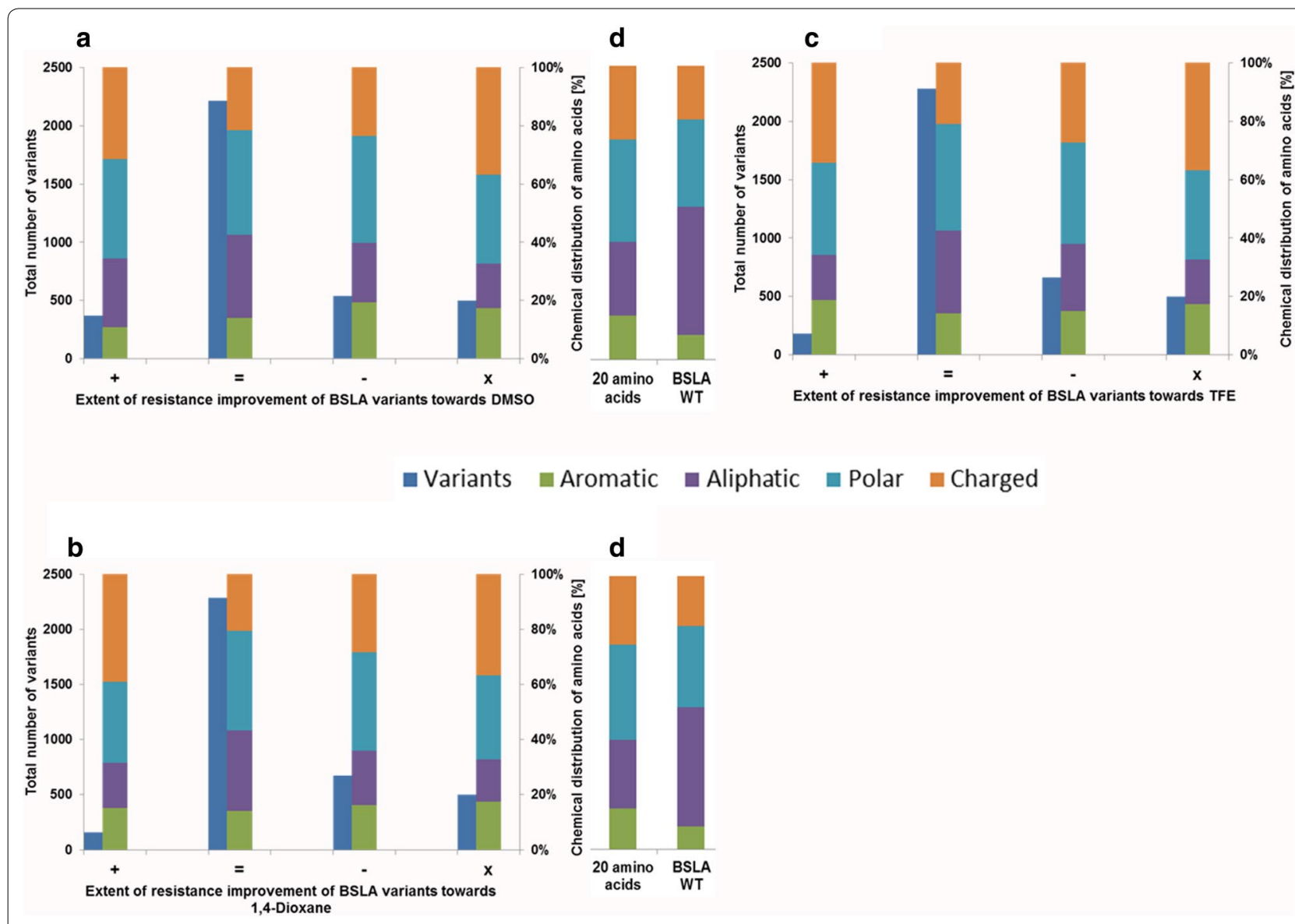

Fig. 3 Number of amino acid substitutions (always the first column) which lead to improved (+), unchanged (=), decreased ( - ) organic cosolvent resistance and inactive variants $(x)$ in $\mathbf{a}$ DMSO, b DOx, and $\mathbf{c T F E}$. $\mathbf{d}$ Distribution of the 20 amino acids according to the genetic code (right) and amino acids composition of WT BSLA (left). Grouped into aromatic ( $F, Y$, and W), aliphatic ( $A, V, L, I$ and $G)$, charged (D, E, H, K and R), and polar (C, M, $P, S, T, N$ and $Q)$. For each organic solvent resistance group $(+,=,-, X)$, the left bar displays the total number of variants, and the right shows the distribution of the amino acids. +: variants with improved resistance; $=$ : unchanged resistance; - : decreased resistance; $\times$ : inactive in buffer

substitutions for all three organic solvents (takes up to $34-41 \%$ of all the beneficial substitution at exposed positions). At buried positions, polar substitutions are more preferred to improve organic solvent resistance (type "+") for all three organic solvents, taking up to $38-44 \%$ of all beneficial substitutions. As previously observed (Frauenkron-Machedjou et al. 2015), the fraction of polar substitutions which resulted in inactive BSLA variants (type " $x$ ") is significantly higher at exposed positions (38\%) than at buried positions (26\%). Notably, charged substitutions at the buried positions resulted in a higher fraction of inactive variants (type " $\times$ "; $41 \%$ ) than the other three types and polar substitutions predominantly reduced activity (type "-"; $32-41 \%$ for all three organic solvents) of BSLA. The amino acids distribution of BSLA WT has more aliphatic amino acids (exposed as well as buried; Additional file 1: Table S10).

\section{Discussion}

Being able to routinely employ enzymes in organic solvents would offer exciting possibilities in biocatalysis for chemical production. Many enzyme classes (e.g. hydrolases, oxidoreductases, transferases, etc.) have been successfully tailored toward improved cosolvent resistance by using protein engineering methodologies (Stepankova et al. 2013; Carrea and Riva 2000). These studies provided initial clues to reengineer focused regions of enzymes (e.g. substrate access tunnel and substrate binding pocket) and to strengthen interactions between amino acids (e.g. introducing disulfide bridges for improved organic cosolvent tolerance).

None of these studies explored nature's full potential to improve the targeted enzymes. In a first systematic study a complete saturation mutagenesis library of BSLA (181 AAs; SSM-BSLA library) was screened in presence of 


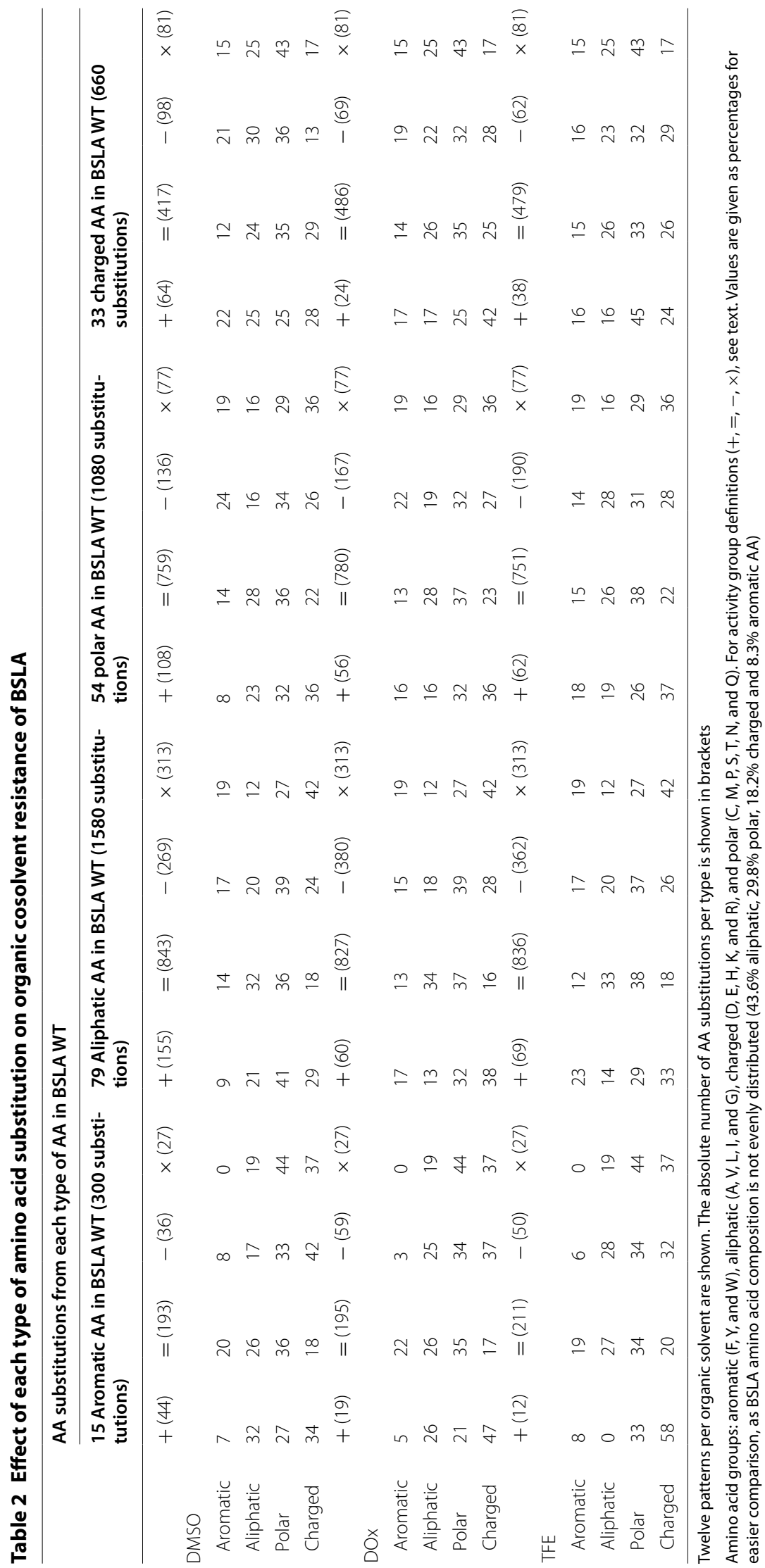


Table 3 Effect of amino acid location (buried, exposed) on organic solvent resistance of BSLA when replacing each type of amino acid in BSLA WT

\begin{tabular}{|c|c|c|c|c|c|c|c|c|}
\hline & \multicolumn{8}{|c|}{ Location of amino acid positions in BSLA (\%) } \\
\hline & \multicolumn{4}{|c|}{ Exposed positions $(128 ; 71 \%)$} & \multicolumn{4}{|c|}{ Buried positions $(53 ; 29 \%)$} \\
\hline & $+(275)$ & $=(1777)$ & $-(319)$ & $\times(189)$ & $+(96)$ & $=(435)$ & $-(220)$ & $\times(309)$ \\
\hline \multicolumn{9}{|l|}{ DMSO } \\
\hline Aromatic & 10 & 15 & 22 & 15 & 14 & 12 & 16 & 19 \\
\hline Aliphatic & 25 & 27 & 21 & 17 & 21 & 36 & 20 & 14 \\
\hline Polar & 31 & 36 & 33 & 38 & 44 & 36 & 41 & 26 \\
\hline \multirow[t]{2}{*}{ Charged } & 35 & 23 & 24 & 30 & 22 & 15 & 23 & 41 \\
\hline & $+(138)$ & $=(1903)$ & $-(330)$ & × (189) & $+(21)$ & $=(385)$ & $-(345)$ & $\times(309)$ \\
\hline \multicolumn{9}{|l|}{ DOx } \\
\hline Aromatic & 16 & 15 & 17 & 15 & 10 & 12 & 15 & 19 \\
\hline Aliphatic & 17 & 27 & 19 & 17 & 14 & 39 & 20 & 14 \\
\hline Polar & 26 & 35 & 35 & 38 & 52 & 40 & 37 & 26 \\
\hline \multirow[t]{2}{*}{ Charged } & 41 & 23 & 28 & 30 & 24 & 9 & 28 & 41 \\
\hline & $+(152)$ & $=(1883)$ & $-(336)$ & × (189) & $+(29)$ & $=(394)$ & $-(328)$ & $\times(309)$ \\
\hline \multicolumn{9}{|l|}{ TFE } \\
\hline Aromatic & 19 & 15 & 15 & 15 & 17 & 12 & 15 & 19 \\
\hline Aliphatic & 16 & 27 & 24 & 17 & 10 & 37 & 22 & 14 \\
\hline Polar & 30 & 36 & 32 & 38 & 38 & 40 & 38 & 26 \\
\hline Charged & 34 & 23 & 29 & 30 & 34 & 11 & 26 & 41 \\
\hline
\end{tabular}

Sixteen patterns per organic solvent are shown. The absolute number of BSLA variants per type is shown in brackets

Amino acid groups: aromatic $(F, Y$, and $W)$, aliphatic $(A, V, L, I$, and $G)$, charged $(D, E, H, K$, and $R)$, and polar $(C, M, P, S, T, N$, and $Q)$. For activity group definitions (,$=$, $-, \times)$ see text. Values are given as percentages for easier comparison, as BSLA amino acid composition is not evenly distributed (43.6\% aliphatic, $29.8 \%$ polar, $18.2 \%$ charged, $8.3 \%$ aromatic). The number of inactive variants $(x)$ was the same for all organic solvents. Column " $\times$ " shows that the number of inactive variants resulted from amino acid substitutions. The activity was measured in a buffer plate which was used as reference (absence of organic solvent) for comparison. Therefore, the number of inactive variants was the same for all four organic solvents

three organic solvents (DMSO, DOx, TFE). Thereby the following questions are addressed: (1) how many single positions can contribute to improved organic cosolvent resistance of BSLA; (2) what types of amino acid substitutions influence and are preferential targets to improve the organic solvent resistance of BSLA; and (3) how does the cosolvent accessibility of amino acid side chains (surface exposed and buried) affect the organic solvent resistance of BSLA.

Overall, a surprising high number of amino acid positions (41-59\%; Fig. 1) was identified to be beneficial for cosolvent resistance of BSLA (DMSO 59\%; DOx 41\%; TFE $41 \%$ ). The latter corresponds to $4.4-10.2 \%$ of all 3620 BSLA variants in the SSM-BSLA library and convincingly explains why cosolvent improved variants can be found after screening of only a few thousand clones in a directed evolution campaign; the obtainable sequence space of BSLA is 20181. Significant differences were found among the tolerance towards the three selected cosolvents, for instance despite that more BSLA variants are found with improved DMSO cosolvent resistance, the number of variants with improvements $>2.5$-fold was 5.7 -fold higher for TFE $(0.17 \%)$ than for DMSO and
DOx $(0.03 \%)$. The higher improvement factors in TFE resistance indicate significant differences in interactions between BSLA and the cosolvents TFE and DMSO/DOx. The latter indicate an unequal potential in optimizing BSLA's tolerance against TFE and DMSO/DOx. Furthermore, the percentage of highly beneficial substitutions (> 2.5-fold improvement) is very low and one can expect that these substitutions would rarely be found in directed evolution campaigns.

The amino acid composition of BSLA (43.6\% aliphatic, $29.8 \%$ polar amino acids, $18.2 \%$ charged, and $8.3 \%$ aromatic) was optimized by nature for its performance in aqueous solution with a preference for aliphatic amino acids. The investigation of individual effect of each type of substitutions when replacing each type of amino acids in BSLA WT showed that improved variants were obtainable by substitution with all four types of amino acids: charged (32-39\%), polar (30-34\%), aliphatic (15-28\%), and aromatic (11-19\%). Obviously charged and polar amino acid substitutions are the predominant beneficial types of substitutions. Both charged and polar amino acids have been reported to contribute to improved organic solvents resistance of enzymes (Park et al. 2012, 
2013; Chen and Arnold 1991; Song and Rhee 2001; Takahashi et al. 2005; Zumarraga et al. 2007), including methanol, ethanol, DMF, 2-propanol, Acetonitrile and DMSO, etc. Postulated reasons were that charged and polar residues could enable the formation of hydrogen bonds between amino acid residues or between surface residues and water molecules which help in maintaining the enzyme structure intact (Park et al. 2012).

Furthermore, substitution of aromatic, polar, charged, and aliphatic amino acid residues with chemically different ones yielded for all three organic solvents more improved variants (68-95\%) than substitutions to chemically similar amino acids. Transversion biased random mutagenesis methods are in respect to transition based methods superior in generating chemically different amino acid substitutions. As a results transversion biased methods will in comparison to transition biased methods reduce screening efforts. A similar preference for transversions was reported for the BSLA resistance towards ionic liquids (Frauenkron-Machedjou et al. 2015) when aromatic/polar/aliphatic amino acids are preferentially replaced by chemical different ones.

What influence has the location of the substitution within BSLA? More beneficial substitutions per positions were obtained at surface-exposed positions than at buried positions (DMSO: 275 vs 96; DOx: 138 vs 21; TFE: 152 vs 29). Computational efforts should therefore preferentially target surface-exposed residues since the probability (the number of possible beneficial substitutions is per position) to identify beneficial positions at exposed positions is higher than at buried positions. Substitutions to charged amino acids on the surface of BSLA (DMSO 35\%; DOx 41\%; TFE 34\%) yielded more improved variants than the other three types of substitutions. This finding applies for all three selected organic solvents. The situation is opposite at buried position of BSLA. At buried positions substitutions to polar amino acids are strongly preferred (DMSO 44\%; DOx $52 \%$; TFE $38 \%$ ) over all three types of substitutions and again the trend is consistent for all three selected organic solvents: (Table 3). The top 10 organic solvent resistant BSLA variants are summarized in Additional file 1: Table S11 and their locations on the structure of BSLA are shown in Additional file 1: Figure. S4. In DMSO, 9 of 10 most beneficial substitutions were polar, suggesting the importance of enhanced interactions (e.g., hydrogen bonds) between substituted residues with surrounding amino acids or waters. Similar trend was observed for the top ten beneficial substitutions towards DOx (8 out of 10 were polar), while only five of ten beneficial substitutions in TFE were polar. Substitutions within BSLA were mostly located in loops (DMSO 44\%; DOx 47\%; TFE 43\%) and on helices (DMSO 34\%; DOx 36\%; TFE 43\%) as described in detail in Additional file 1: Table S6. In summary, loop regions represent a preferred targeting location for improving solvent tolerance against DMSO, DOx, and TFE. A role of substitutions in loop regions to improve cosolvent resistance has previously been reported (Wintrode and Arnold 2001). Substitutions of surface located residues were in many cases reported to contribute to organic solvent resistance of enzymes (Park et al. 2012, 2013; Kawata and Ogino 2009; Martinez and Arnold 1991; Martinez et al. 1992). Ogino et al. (2007) speculated that charged substitutions on the surface prevent organic solvents molecules to diffuse into the protease from Pseudomonas aeruginosa PST-01. Yedavalli and Rao (2013) performed site saturation mutagenesis (SSM) of all the amino acid positions in the loops of a BSLA to find DMSO resistant BSLA variants. In contrast to our study, six beneficial positions were identified and recombined, resulting in a final variant $6 \mathrm{SR}$ with 8-fold higher catalytic turnover in $60 \mathrm{vol} \%$ DMSO. Three of the six mutations increased the surface polarity suggesting that substitutions with more polar amino acids may be helpful to improve the stability of a protein in a water-miscible organic solvent.

\section{Conclusions}

A surprisingly high number of amino acid positions contribute to the improvements in organic solvent resistance of BSLA. Substitutions to charged amino acids on the surface and to polar amino acids at buried positions are the main beneficiaries. Substitutions to chemically different amino acids led to significantly more BSLA variants with improved organic cosolvent resistance than substitutions to chemically similar ones. Significant differences were observed in the evolutionary potential in respect to number of beneficial positions and extend of improvements were found among DMSO, DOx and TFE. Followup studies have to confirm if the discovered trends can be transferred to the industrially important enzymes' classes.

\section{Additional file}

Additional file 1. Supplymentary information.

\section{Abbreviations}

WT: wild type; EV: empty vector; R: residual activity; BSLA: Bacillus subtilis lipase A; TFE: 2,2,2-trifluoroethanol; DOx: 1,4-dioxane; DMSO: dimethyl sulfoxide; SSM: site saturation mutagenesis; $p N P B$ : $p$-nitrophenyl butyrate.

\section{Authors' contributions}

VJF-M, KEJ, US and LZ conceived and designed the research; VJF-M, AF and LW performed the experiments; VJF-M and LZ analyzed the data; VJF-M, JZ, US and $L Z$ wrote the paper. All authors read and approved the final manuscript.

\section{Author details}

${ }^{1}$ Lehrstuhl für Biotechnologie, RWTH Aachen University, Worringerweg 3, 52074 Aachen, Germany. ${ }^{2}$ Tianjin Institute of Industrial Biotechnology, 
Chinese Academy of Sciences, West 7th Avenue 32, Tianjin 300308, China. ${ }^{3}$ DWI an der RWTH Aachen eV, RWTH Aachen University, Forckenbeckstrasse 50, 52056 Aachen, Germany. ${ }^{4}$ Institut für Molekulare Enzymtechnologie, Heinrich Heine Universität Düsseldorf, Wilhelm-Johnen Strasse, 52426 Jülich, Germany. ${ }^{5}$ Institut für Molekulare Enzymtechnologie, Forschung Zentrum Jülich GmbH, Wilhelm-Johnen Strasse, 52426 Jülich, Germany.

\section{Acknowledgements}

Not applicable.

\section{Competing interests}

The authors declare that the research was conducted in the absence of any commercial or financial relationships that could be construed as a potential competing interests.

\section{Availability of data and materials}

All data generated or analyzed during this study are included in the main manuscript file and additional file.

\section{Consent for publication}

The authors approved the consent for publishing the manuscript.

\section{Ethics approval and consent to participate}

All the authors have read and agreed the ethics for publishing the manuscript.

\section{Funding}

The authors wish to thank the Deutsche Forschungsgemeinschaft (DFG) for financial support through the Research Training Group "BioNoCo-Biocatalysis using non-conventional media" (GRK 1166) and the Hundred Talents Program of Chinese Academy of Sciences to Prof. Leilei Zhu.

\section{Publisher's Note}

Springer Nature remains neutral with regard to jurisdictional claims in published maps and institutional affiliations.

Received: 25 October 2017 Accepted: 22 December 2017

Published online: 06 January 2018

\section{References}

Arnold FH (1990) Engineering enzymes for non-aqueous solvents. Trends Biotechnol 8:244-249

Arpigny JL, Jaeger KE (1999) Bacterial lipolytic enzymes: classification and properties. Biochem J 343:177-183

Barettino D, Feigenbutz M, Valcarcel R, Stunnenberg HG (1994) Improved method for PCR-mediated site-directed mutagenesis. Nucleic Acids Res 22(3):541-542

Bustos-Jaimes I, Mora-Lugo R, Calcagno ML, Farres A (2010) Kinetic studies of Gly28: ser mutant form of Bacillus pumilus lipase: changes in k(cat) and thermal dependence. Biochim Biophys Acta-Proteins Proteom 1804(12):2222-2227

Carrea G, Riva S (2000) Properties and synthetic applications of enzymes in organic solvents. Angew Chemie Int Ed 39(13):2226-2254

Castro GR, Knubovets T (2003) Homogeneous biocatalysis in organic solvents and water-organic mixtures. Crit Rev Biotechnol 23(3):195-231

Chen KQ, Arnold FH (1991) Enzyme engineering for nonaqueous solventsrandom mutagenesis to enhance activity of subtilisin-E in polar organic media. Bio-Technology 9(11):1073-1077

Chothia C (1976) Nature of accessible and buried surfaces in proteins. J Mol Biol 105(1):1-14

Frauenkron-Machedjou VJ, Fulton A, Zhu L, Anker C, Bocola M, Jaeger KE, Schwaneberg U (2015) Towards understanding directed evolution: more than half of all amino acid positions contribute to ionic liquid resistance of Bacillus subtilis lipase A. ChemBioChem 16(6):937-945

Fulton A, Frauenkron-Machedjou VJ, Skoczinski P, Wilhelm S, Zhu L, Schwaneberg U, Jaeger KE (2015) Exploring the protein stability landscape: Bacillus subtilis lipase A as a model for detergent tolerance. ChemBioChem 16(6):930-936
Gorman LAS, Dordick JS (1992) Organic solvents strip water off enzymes. Biotechnol Bioeng 39(4):392-397

Kawata T, Ogino H (2009) Enhancement of the organic solvent-stability of the LST-03 lipase by directed evolution. Biotechnol Prog 25(6):1605-1611

Kawata T, Ogino H (2010) Amino acid residues involved in organic solvent-stability of the LST-03 lipase. Biochem Biophys Res Commun 400(3):384-388

Koudelakova T, Chaloupkova R, Brezovsky J, Prokop Z, Sebestova E, Hesseler M, Khabiri M, Plevaka M, Kulik D, Kuta Smatanova I (2013) Engineering enzyme stability and resistance to an organic cosolvent by modification of residues in the access tunnel. Angew Chem Int Ed 52(7):1959-1963

Li C, Tan T, Zhang H, Feng W (2010) Analysis of the conformational stability and activity of Candida antarctica lipase B in organic solvents: insight from molecular dynamics and quantum mechanics/simulations. J Biol Chem 285(37):28434-28441

Liu S, Fang Y, Xu W, Lu M, Wang S, Chen L (2009) Screening and identification of a novel organic solvent-stable lipase producer. Ann Microbiol 59(3):539-543

Liu HF, Zhu L, Bocola M, Chen N, Spiess AC, Schwaneberg U (2013) Directed laccase evolution for improved ionic liquid resistance. Green Chem 15(5):1348-1355

Lynch M (2010) Evolution of the mutation rate. Trends Genet 26(8):345-352

Markel U, Zhu L, Frauenkron-Machedjou VJ, Zhao J, Bocola M, Davari MD, Jaeger KE, Schwaneberg U (2017) Are directed evolution approaches efficient in exploring nature's potential to stabilize a lipase in organic cosolvents? Catalysts 7(5):142

Martinez P, Arnold FH (1991) Surface charge substitutions increase the stability of. alpha.-lytic protease in organic solvents. J Am Chem Soc 113(16):6336-6337

Martinez P, Van Dam ME, Robinson AC, Chen K, Arnold FH (1992) Stabilization of subtilisin $\mathrm{E}$ in organic solvents by site-directed mutagenesis. Biotechnol Bioeng 39(2):141-147

Micaelo NM, Soares CM (2007) Modeling hydration mechanisms of enzymes in nonpolar and polar organic solvents. FEBS J 274(9):2424-2436

Ogino H, Ishikawa H (2001) Enzymes which are stable in the presence of organic solvents. J Biosci Bioeng 91 (2):109-116

Ogino H, Uchiho T, Doukyu N, Yasuda M, Ishimi K, Ishikawa H (2007) Effect of exchange of amino acid residues of the surface region of the PST-01 protease on its organic solvent-stability. Biochem Biophys Res Commun 358(4):1028-1033

Park HJ, Joo JC, Park K, Yoo YJ (2012) Stabilization of Candida antarctica lipase $B$ in hydrophilic organic solvent by rational design of hydrogen bond. Biotechnol Bioprocess Eng 17(4):722-728

Park HJ, Joo JC, Park K, Kim YH, Yoo YJ (2013) Prediction of the solvent affecting site and the computational design of stable Candida antarctica lipase B in a hydrophilic organic solvent. J Biotechnol 163(3):346-352

Roccatano D, Wong TS, Schwaneberg U, Zacharias M (2006) Toward understanding the inactivation mechanism of monooxygenase P450 BM-3 by organic cosolvents: a molecular dynamics simulation study. Biopolymers 83(5):467-476

Shirai K, Jackson RL, Quinn DM (1982) Reciprocal effect of apolipoproteinC-li on the lipoprotein lipase-catalyzed hydrolysis of para-nitrophenyl butyrate and trioleoylglycerol. J Biol Chem 257(17):200-203

Song JK, Rhee JS (2001) Enhancement of stability and activity of phospholipase A(1) in organic solvents by directed evolution. BBA-Protein Struct Mol 1547(2):370-378

Stepankova V, Bidmanova S, Koudelakova T, Prokop Z, Chaloupkova R, Damborsky J (2013) Strategies for stabilization of enzymes in organic solvents. Acs Catal 3(12):2823-2836

Takahashi T, Ng KKS, Oyama H, Oda K (2005) Molecular cloning of the gene encoding vibrio metalloproteinase vimelysin and isolation of a mutant with high stability in organic solvents. J Biochem 138(6):701-710

van Pouderoyen G, Eggert T, Jaeger KE, Dijkstra BW (2001) The crystal structure of Bacillus subtilis lipase: a minimal alpha/beta hydrolase fold enzyme. J Mol Biol 309(1):215-226

Verma R, Schwaneberg U, Roccatano D (2014) Insight into the redox partner interaction mechanism in cytochrome P450BM-3 using molecular dynamics simulations. Biopolymers 101(3):197-209

Wintrode PL, Arnold PH (2001) Temperature adaptation of enzymes: lessons from laboratory evolution. Adv Protein Chem 55:161-225 
Wong TS, Arnold FH, Schwaneberg U (2004) Laboratory evolution of cytochrome P450 BM-3 monooxygenase for organic cosolvents. Biotechnol Bioeng 85(3):351-358

Wong TS, Roccatano D, Zacharias M, Schwaneberg U (2006) A statistical analysis of random mutagenesis methods used for directed protein evolution. J Mol Biol 355(4):858-871

Wong TS, Roccatano D, Schwaneberg U (2007) Are transversion mutations better? A Mutagenesis Assistant Program analysis on P450 BM-3 heme domain. Biotechnol J 2(1):133-142

Yang L, Dordick JS, Garde S (2004) Hydration of enzyme in nonaqueous media is consistent with solvent dependence of its activity. Biophys $J$ 87(2):812-821
Yedavalli P, Rao NM (2013) Engineering the loops in a lipase for stability in DMSO. Protein Eng Des Sel 26(4):317-324

Zaks A, Klibanov AM (1988) The effect of water on enzyme action in organic media. J Biol Chem 263(17):8017-8021

Zhao J, Jia N, Jaeger KE, Bocola M, Schwaneberg U (2015) lonic liquid activated Bacillus subtilis lipase A variants through cooperative surface substitutions. Biotechnol Bioeng 112(10):1997-2004

Zumarraga M, Bulter T, Shleev S, Polaina J, Martinez-Arias A, Plow FJ, Ballesteros A, Alcalde M (2007) In vitro evolution of a fungal laccase in high concentrations of organic cosolvents. Chem Biol 14(9):1052-1064

\section{Submit your manuscript to a SpringerOpen ${ }^{\circ}$ journal and benefit from:}

- Convenient online submission

- Rigorous peer review

- Open access: articles freely available online

- High visibility within the field

- Retaining the copyright to your article

Submit your next manuscript at $\boldsymbol{\nabla}$ springeropen.com 